\title{
$\underline{\mathbf{P}-5}$
}

\section{Effect of Moisture Content on Physical Characteristics of Orthosiphon Stamineus (Benth) Leaf Water Extract Powder}

\author{
Mahibub Kanakal ${ }^{1,2,3, *}$, Amin Abdul Majid ${ }^{2, *}$, Aman Abdul Majid ${ }^{3}$, Munavvar Sattar ${ }^{2}$, Noor Shahida \\ $\mathrm{Ajmi}^{4}$ and Maryam Farooqui \\ ${ }^{I}$ Department of Pharmaceutics, Faculty of Pharmacy, Universiti Teknologi MARA, Bertam Campus, Kepala Batas, \\ 13200, P.Pinang,Malaysia; ${ }^{2}$ School of Pharmaceutical Sciences, Universiti Sains Malaysia, 13200, P.Pinang, Malaysia; \\ ${ }^{3}$ Cluster of Integrative Medicine, Advanced Medical and Dental Institute, Universiti Sains Malaysia, Kepala Batas, \\ 13200, P.Pinang, Malaysia; ${ }^{4}$ Department of Pharmacy, Allianze University College of Medical Sciences (AUCMS), \\ 13200, Kepala Batas, P.Pinang, Malaysia; E-mail: mehboob@ppinang.uitm.edu.my
}

Water based plant extract powders are commonly used for various medicinal applications due to their simple method of extraction. These extract powders are usually hygroscopic which make them difficult to handle especially in high humidity environment. Hence to further develop them into various pharmaceutical formulations becomes a challenge. This work looks into hygroscopic extract formulation in granular form which can reduce moisture content and increase flow. Water based leaf extract of Orthosiphon Stamineus (Benth) was selected for this study. Extract powder and formulated granules were stored at $30^{\circ} \mathrm{C} / 65 \%$ and $40^{\circ} \mathrm{C} / 75 \% \mathrm{RH}$ for three months. Influence of excipients on formulation was tested in terms of moisture content, color, bulk density, tap density, compression index, and flow properties. Formulation with Magnesium Aluminometasilicate reduced the moisture content and increase the flow during and after storage for predetermined time. Where as other selected excipients decrease moisture content without improving the flow. The findings provide an insight into a possible approach for formulating moisture-sensitive pharmaceutical products, especially dry powder preparations for use in high humidity environment.

Keywords: Moisture content, Physical Chareteristics, Water extract. 